Revue des patrimoines

$21 \mid 2013$

De l'art de bâtir aux champs à la ferme moderne

\title{
Le débat sur l'architecture rurale en Italie et l'exposition de Giuseppe Pagano à la Triennale de 1936
}

Giovanna D'Amia

\section{OpenEdition}

Journals

Édition électronique

URL : http://journals.openedition.org/insitu/10454

DOI : 10.4000/insitu. 10454

ISSN : 1630-7305

Éditeur

Ministère de la culture

Référence électronique

Giovanna D'Amia, « Le débat sur l'architecture rurale en Italie et l'exposition de Giuseppe Pagano à la Triennale de 1936 », In Situ [En ligne], 21 | 2013, mis en ligne le 12 juillet 2013, consulté le 01 mai 2019. URL : http://journals.openedition.org/insitu/10454; DOI : 10.4000/insitu.10454

Ce document a été généré automatiquement le 1 mai 2019.

\section{(c)}

In Situ Revues des patrimoines est mis à disposition selon les termes de la licence Creative Commons Attribution - Pas d'Utilisation Commerciale - Pas de Modification 4.0 International. 


\title{
Le débat sur l'architecture rurale en Italie et l'exposition de Giuseppe Pagano à la Triennale de 1936
}

\author{
Giovanna D'Amia
}

\section{Architecture rurale ou rustique ? Le débat interdisciplinaire ${ }^{1}$}

1 Le débat sur l'architecture rurale en Italie durant l'entre-deux-guerres s'ancre dans les courants européens visant l'étude des relations entre l'homme et l'environnement qui, dans la seconde moitié du XIX ${ }^{e}$ siècle, avaient donné naissance à la "géographie humaine » comme discipline. Ce sont en effet les géographes qui s'en occupèrent les premiers; depuis la tribune que leur offrait la Rivista geografica italiana (Revue géographique italienne), ils tournèrent leur attention vers le débat international et l'expérience française des Annales de géographie de Paul Vidal de la Blache².

2 La maison rurale, comprise comme élément de médiation entre l'homme et l'environnement, prit une position centrale en tant qu'objet d'étude à partir du début des années 1920, dans le sillage des enquêtes lancées en France par Albert Demangeon. Celuici proposait une classification typologique des demeures rurales en partant des rapports entre le logis et les espaces dédiés aux fonctions agricoles ${ }^{3}$. À ses yeux, en effet, la maison est surtout un "instrument de travail» et elle doit être classée d'après les fonctions qu'elle abrite et des opérations qu'elle rend possible. Le travail de Demangeon servit de point de départ à Renato Biasutti, membre de la Commission d'étude sur l'habitation rurale issue du Congrès international de géographie organisé au Caire en 1925. Celui-ci se fit le promoteur d'un outil méthodologique conçu à partir du cas italien et du lancement d'une enquête systématique sur les différentes régions de la Péninsule ${ }^{4}$. Bien qu'elle confirme la classification proposée par le géographe français, la méthode d'enquête de Biasutti - synthétisée dans le questionnaire présenté au $\mathrm{X}^{\mathrm{e}}$ Congrès géographique italien 
de 1927 - se révélait pourtant plus sensible aux particularités des populations et à la connaissance des caractéristiques régionales ${ }^{5}$.

La contribution des géographes s'enrichit de l'intervention de Gina Algranati, qui permit la confrontation avec les ethnographes et qui suggéra une classification tenant également compte des matériaux utilisés et de la topographie ${ }^{6}$. La position de Bruno Nice défendant l'importance de l'étude d'éléments non structuraux, comme la répartition intérieure des pièces de l'habitation, joua aussi un rôle. Ce dernier considérait que la classification structurale de Biasutti s'avérait peu adéquate pour des territoires marqués par de fortes influences ethniques, comme c'est le cas pour la Vénétie julienne ${ }^{7}$.

4 La prise en compte du poids spécifique du facteur ethnique dans la classification des demeures rurales donna une plus grande importance à la rencontre avec les ethnographes qui avaient commencé à s'occuper de l'architecture « rustique » (c'est la formule la plus fréquente dans leurs études) dans le cadre des recherches sur les expressions artistiques de la culture populaire ${ }^{8}$. À la différence des géographes qui se focalisaient sur les rapports entre architecture et économie agricole, les ethnographes privilégiaient les aspects formels et décoratifs et mettaient en avant un plus grand intérêt pour la dimension historique de l'habitation rurale, entendue comme une manifestation culturelle en transformation. À l'occasion du III ${ }^{\mathrm{e}}$ Congrès des arts et traditions populaires, qui se tint dans la ville de Trente en 1934, Giulio Brocherel proposa une étude comparée de la maison rurale fondée sur l'analyse de sa structure interne et sur l'examen des éléments de construction, afin d'en reconstruire l'évolution à partir de sa forme primordiale 9 . Pour sa part, le Comité pour les arts populaires mit au point en 1936 son propre questionnaire pour le recueil d'informations sur les «objets et les documents d'architecture $»^{10}$.

5 L'intérêt pour les aspects formels propre aux ethnographes était partagé par les artistes et les architectes qui adoptèrent, en général, l'expression de "casa rustica " (maison rustique) et qui intervenaient de manière assidue dans les pages de la revue Lares $^{11}$. Si les artistes proposaient de leur côté une mise en valeur du paysage rural, les architectes démontraient une sensibilité plus grande pour les procédés de construction et une conscience précoce de l'importance de l'architecture «mineure » et de la nécessité de sa conservation. 
Figure 1

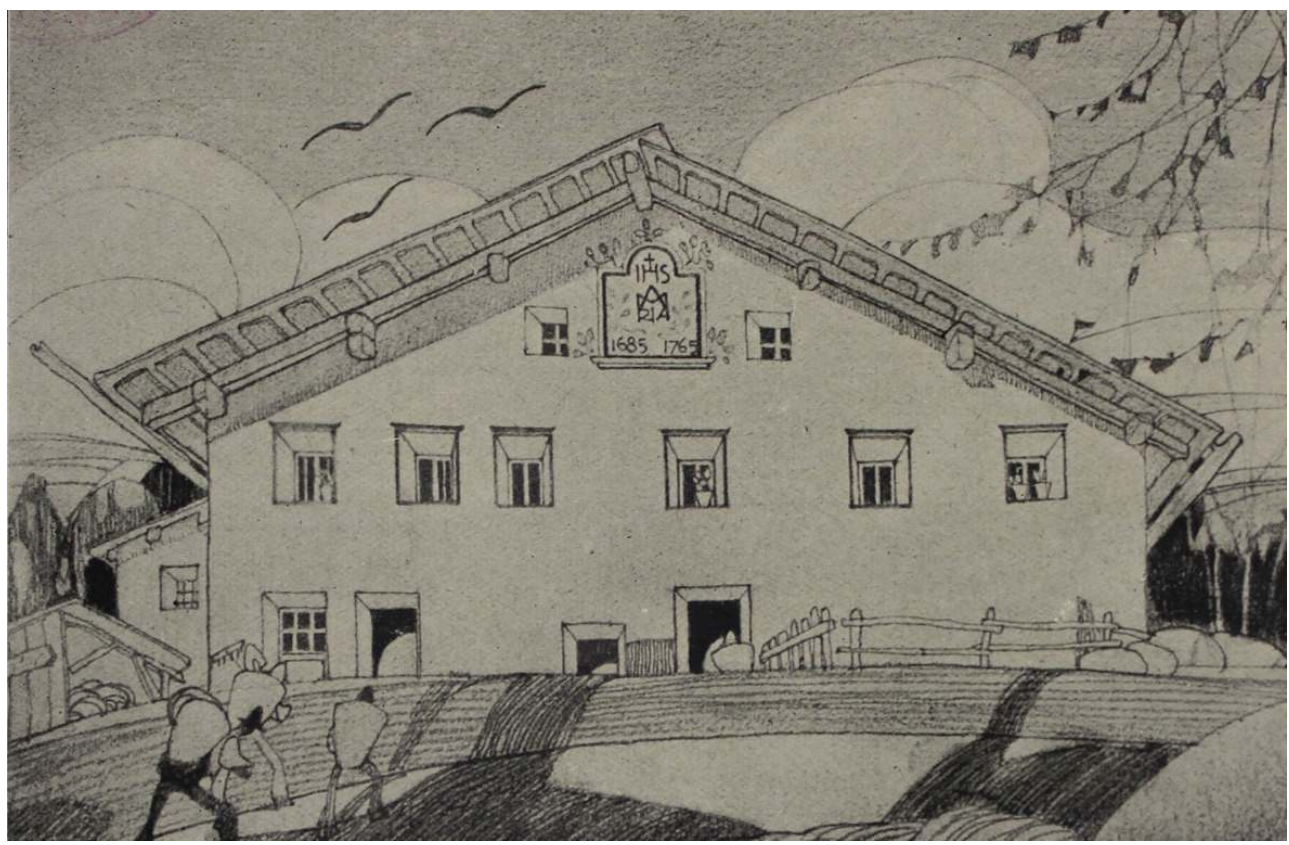

Enrico Griffini, Maison rurale de la région de Trente. D'après Architettura e arti decorative, IV, 1924-25, vol. II.

REPRO. D'AMIA, GIOVANNA. (C) GIOVANNA D'AMIA.

Figure 2

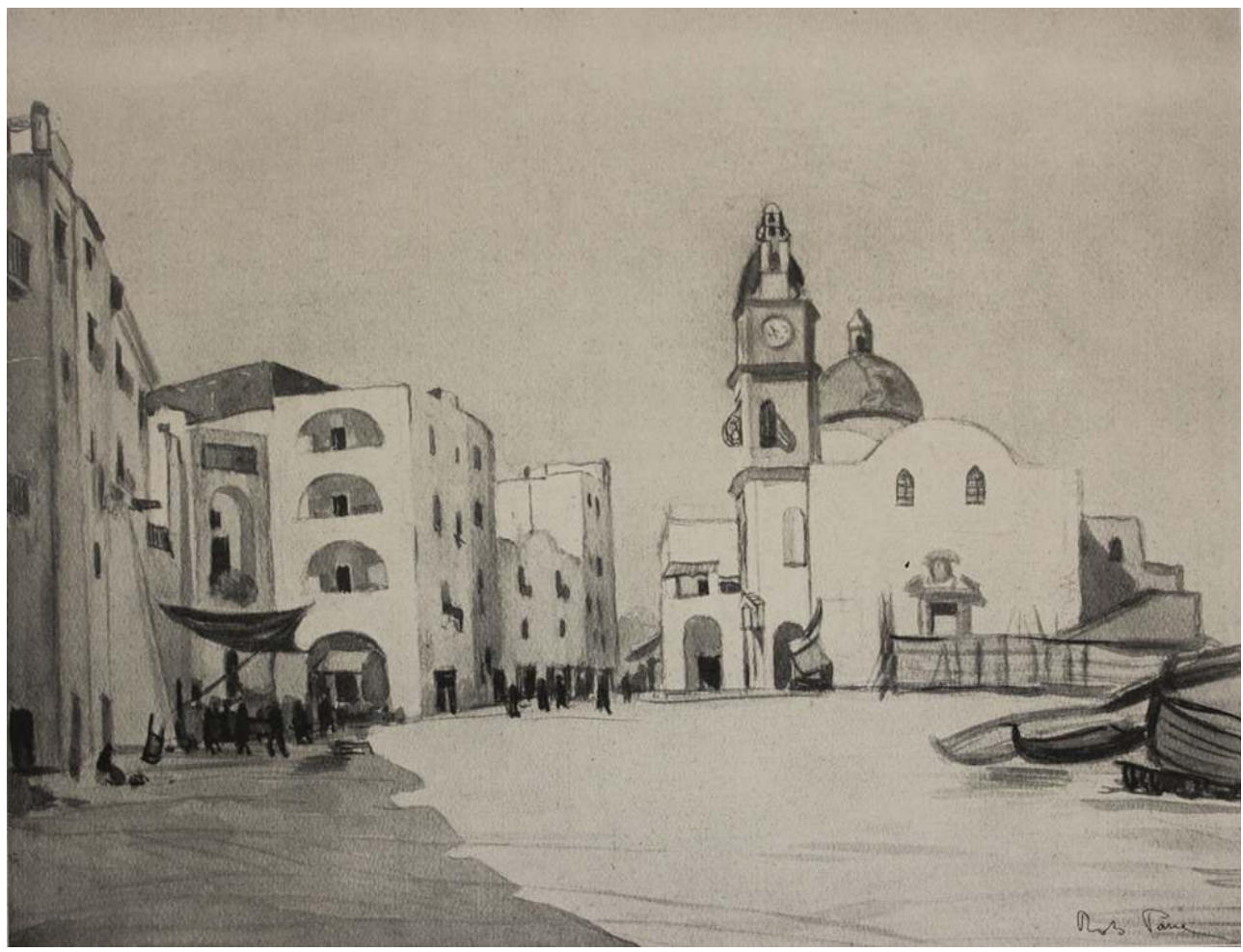

Roberto Pane, Marine à Procida. D'après Architettura e arti decorative, VII, 1927-1928, vol. II. REPRO. D'AMIA, GIOVANNA. (c) GIOVANNA D'AMIA. 
Figure 3

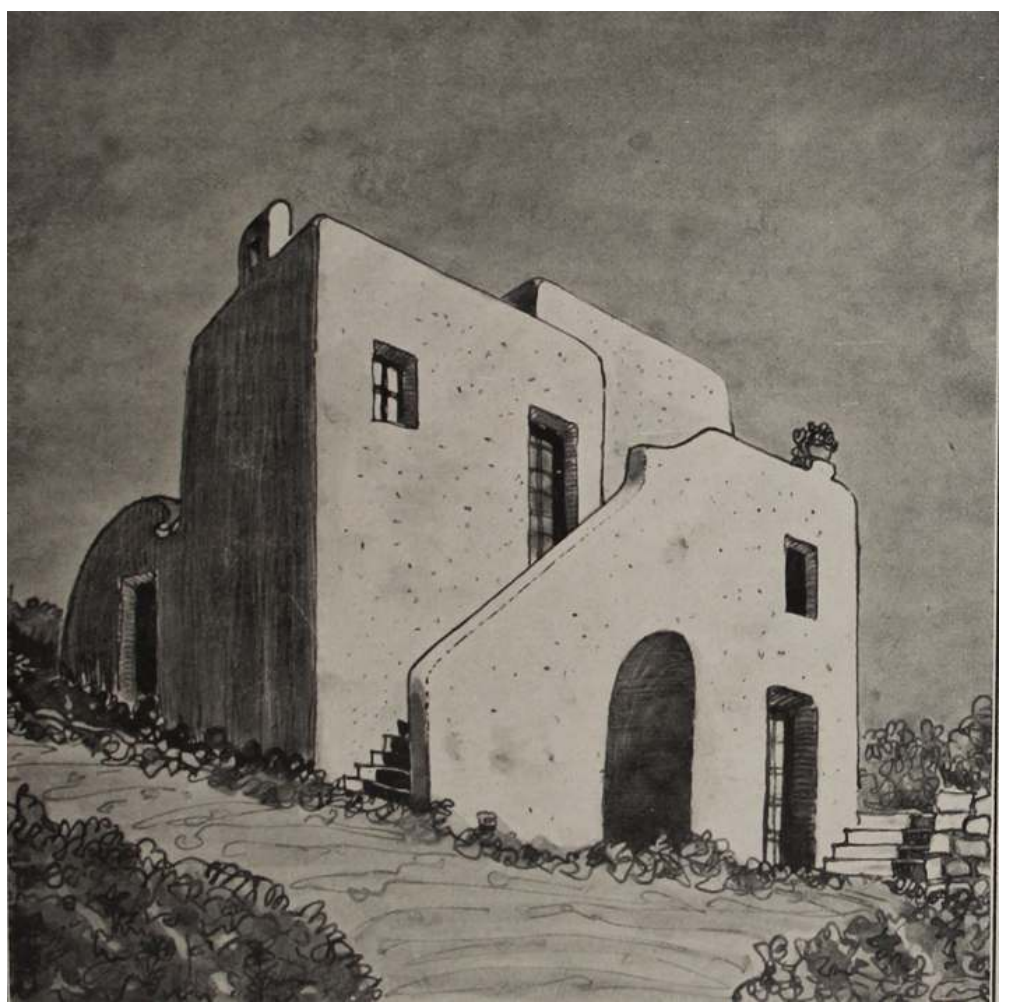

Plinio Marconi, Maison à Capri. D'après Architettura e arti decorative, IX, 1929-1930, vol. I.

REPRO. D'AMIA, GIOVANNA. (c) GIOVANNA D'AMIA.

Le débat lancé dans les milieux de la culture architecturale passe par la participation des principales revues de ce domaine. Les voix d'Enrico Griffini et de Giuseppe Gerola s'exprimaient dans les pages de Architettura e arti decorative (Architecture et arts décoratifs) en appelant l'attention sur la simplicité des demeures de la région de Trente et du Haut-Adige ${ }^{12}$ (fig. $\mathbf{n}^{\circ} \mathbf{1}$ ). Roberto Pane et Plinio Marconi, pour leur part, dépassaient l'optique de la seule description pour enquêter sur les procédés de construction des maisons de Capri et de la région du Vésuve (fig. $n^{\circ} 2, n^{\circ} 3$ ), tout en s'interrogeant sur leurs origines $^{13}$. Lors d'une intervention dans Rassegna di Architettura, Arduino Berlam, analysant les maisons caractéristiques de la Carnia, y trouvait « une solide expérience et une adéquation effective aux besoins ${ }^{14} »$, tandis que dans Casabella, Mario Tinti condamnait « la mode de l'art rustique », en assimilant les maisons rurales à un modèle de construction étranger à la rhétorique des styles ${ }^{15}$. De même, dans les pages de Domus, Carlo Enrico Rava reconnaissait dans l'architecture autochtone, à Capri comme en Lybie, un usage conscient des matériaux et une adaptation aux conditions climatiques ${ }^{16}$, tandis que Giovanni Michelucci proposait certaines maisons toscanes comme « des sources de l'architecture italienne moderne ${ }^{17} »\left(\right.$ fig. $\left.\mathbf{n}^{\circ} \mathbf{4}\right)$. 
Figure 4

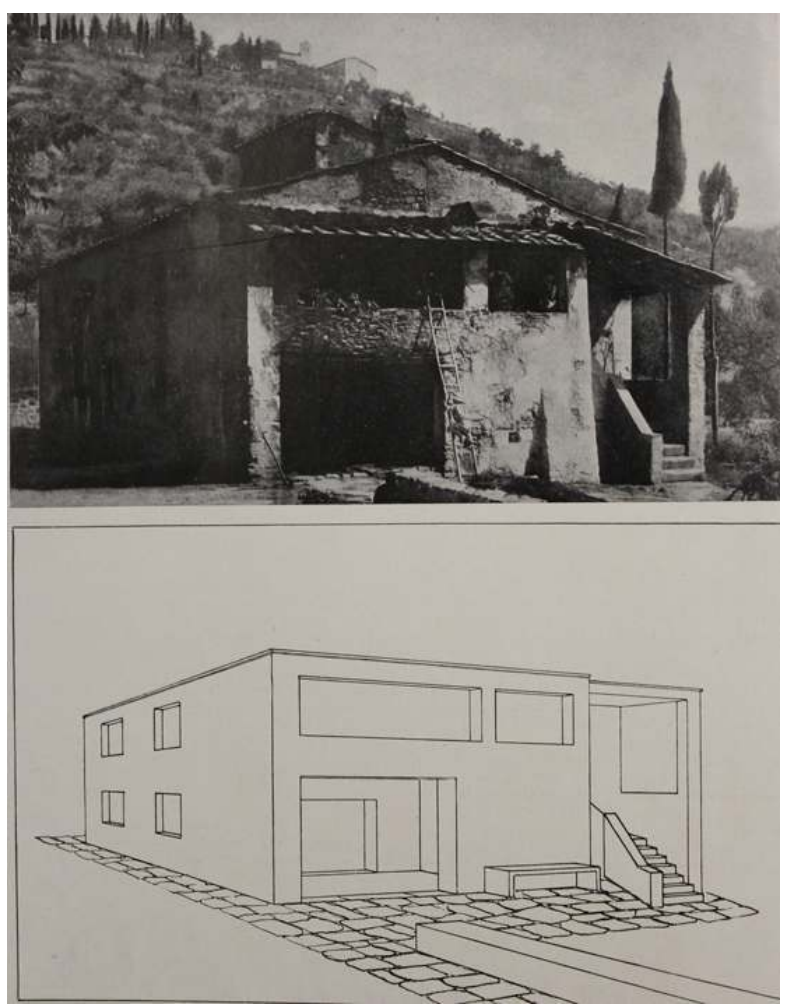

Giovanni Michelucci, Maison de la région du Chianti en Toscane. D’après Domus, 1932, n 56.

REPRO. D'AMIA, GIOVANNA. () GIOVANNA D'AMIA.

En résumé, dans les années qui précédèrent la Triennale de 1936, la maison rurale fit l'objet de l'attention des architectes traditionnalistes comme des partisans de l'architecture moderne: les premiers (Gerola et Berlam, entre autres) la concevaient comme un patrimoine de formes servant de source d'inspiration dans la recherche d'une architecture typiquement nationale; les seconds (comme Marconi, Rava et Michelucci) considéraient qu'elle anticipait sur les nouvelles tendances par son absence de décoration, par la sincérité dans la construction et sa pertinence vis-à-vis des exigences fonctionnelles. Dans tous les cas, dans la réflexion des architectes, l'étude de l'architecture rurale privilégiait le langage formel ainsi que les valeurs esthétiques, qui n'étaient pas sans conséquence en termes de projets.

Dans certains cas, la réflexion sur la maison rurale se mêlait au débat sur l'architecture méditerranéenne qui, dans le sillage des interventions de Carlo Enrico Rava, entendait construire une voie italienne du rationalisme à partir de la tradition architecturale des peuples latins. On en trouve un exemple éloquent avec l'intervention d'Enrico Peressutti dans les pages de Quadrante. Il reconnaissait dans les «rectangles blancs, simples » et dans « les architectures du vide et du plein » des maisons méditerranéennes (fig. $\mathbf{n}^{\circ} \mathbf{5}$ ) un patrimoine typiquement italien, « découvert par Gropius et Le Corbusier [et] déguisé en nouveauté nordique, comme une invention du $\mathrm{Xx}^{\mathrm{e}}$ siècle $^{18} »$. 
Figure 5

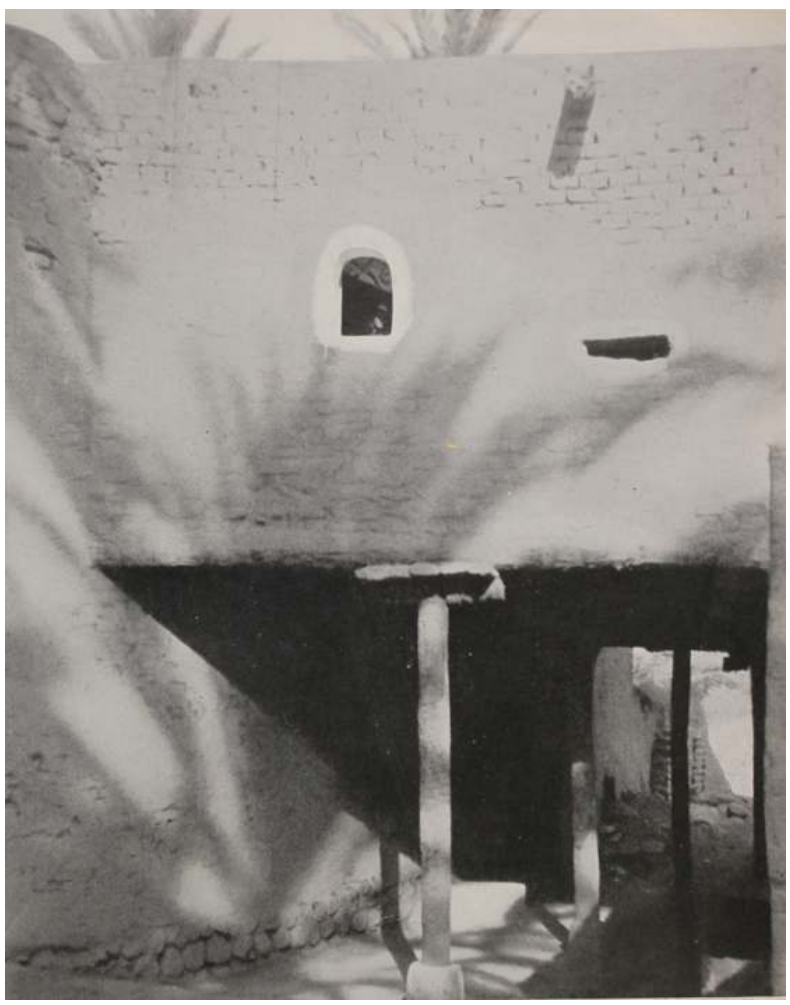

Maison à Biskra. Photographie de E. Galassi. D'après Quadrante, 1935, nº 21.

REPRO. D'AMIA, GIOVANNA. () GIOVANNA D'AMIA.

La nécessité de mettre au point de nouveaux modèles de fermes contribua également à promouvoir le débat sur l'architecture rurale, et cela dans le cadre de la politique de réorganisation agricole défendue par le régime fasciste, dans la mère patrie comme dans les colonies ${ }^{19}$. Dans ce cas, ce furent surtout les ingénieurs et les techniciens agraires, intéressés essentiellement par les aspects liés à la construction, à l'hygiène et à la gestion, qui abordèrent le thème à l'occasion de congrès ou de concours, ou bien encore dans les revues spécialisées ${ }^{20}$. En ce qui concerne les contributions des architectes dans ce domaine, il faut signaler le modèle de maison rurale publié dans Quadrante par Gaetano Ciocca en 1935 (fig. $\mathbf{n}^{\circ} \mathbf{6}$ ), qui s'opposait à l'insalubrité de la maison traditionnelle avec une construction tout à fait moderne ayant largement recours aux éléments préfabriqués ${ }^{21}$; ou encore la proposition de Gino Levi Montalcini présentée par Giuseppe Pagano dans Casabella (fig. $\mathbf{n}^{\circ} 7$ ), qui remonte aussi à l'année $1935^{22}$. Mais, en général, les architectes tendaient à se concentrer sur les aspects d'organisation et de décoration des parties du logement, en négligeant la conception d'éléments fonctionnels tels que les étables, les granges, les remises et les greniers. 
Figure 6

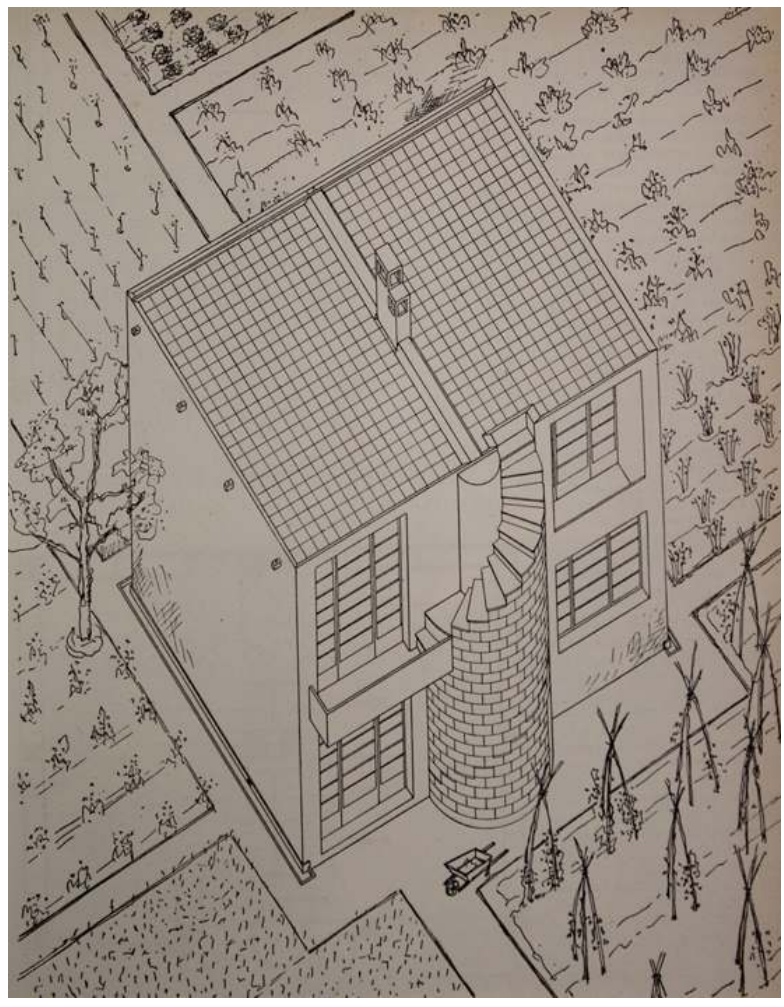

Gaetano Ciocca, Projet de maison rurale. D'après Quadrante, 1935, nº 26. REPRO. D'AMIA, GIOVANNA. () GIOVANNA D'AMIA. 


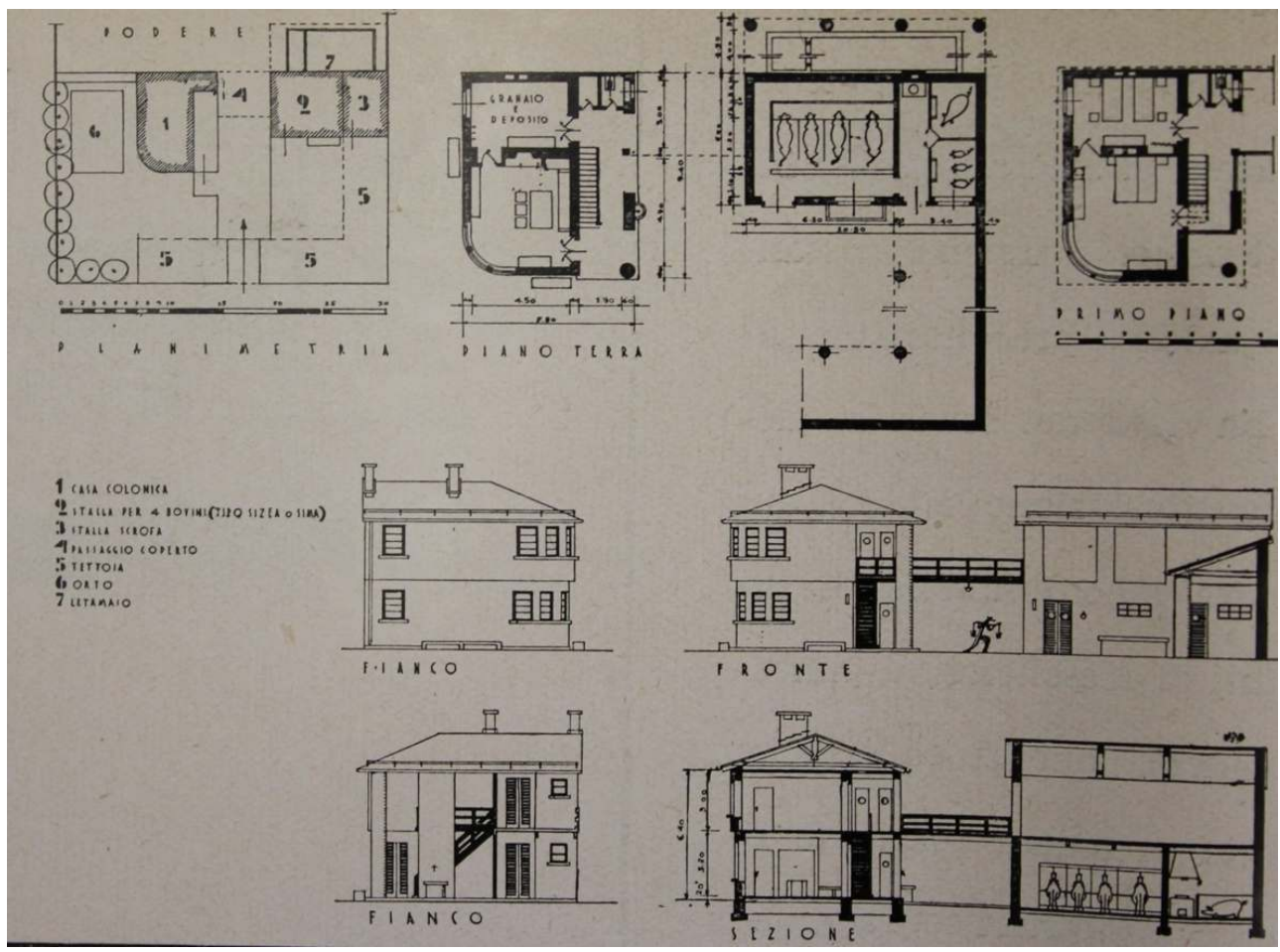

Gino Levi Montalcini, Projet de petite ferme agricole. D’après Casabella, 1935, nº 86.

REPRO. D'AMIA, GIOVANNA. () GIOVANNA D'AMIA.

\section{Giuseppe Pagano et l'exposition de 1936}

10 L'intérêt de Giuseppe Pagano pour l'architecture rurale trouva une première concrétisation dans certains articles publiés dans Casabella, la revue dont il était directeur dès $1933^{23}$ (fig. $\mathbf{n}^{\circ} \mathbf{8}$ ). Dans le fascicule de février - où l'on présentait des projets récents de maisons paysannes dans le cadre de la politique de ruralisation lancée par le régime -, il visait à attirer l'attention des architectes sur un thème qui nécessite « une observance constante et rigoureuse des principes fonctionnels, imposés par les habitudes, par le lieu, par les contraintes climatiques, et par les nécessités techniques imposées par le type de culture $^{24} »$. Bien qu'il ne cite pas ouvertement ses propres sources, Pagano se montre en phase avec les lignes générales du débat de l'époque, en empruntant aux géographes la définition de la maison rurale comme «instrument de travail» et en tirant son inspiration des ethnographes lorsqu'il se réfère aux «habitudes » et à la dimension culturelle du phénomène. 
Figure 8

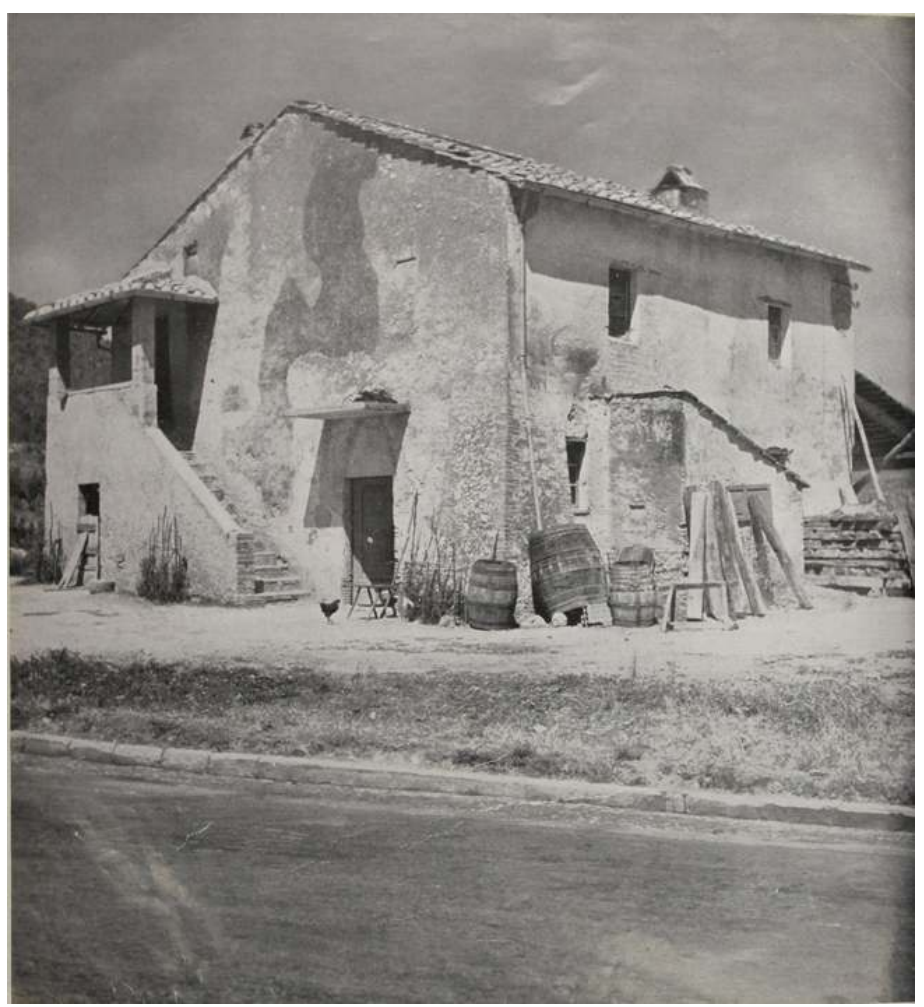

Maison rurale aux environs d'Otricoli en Latium. Photographie de Giuseppe Pagano. D'après Casabella, $1935, n^{\circ} 96$

REPRO. D'AMIA, GIOVANNA. (c) GIOVANNA D'AMIA.

11 Une intervention suivante dans le numéro de novembre trahit les motivations profondes qui amenèrent Pagano à s'occuper des maisons rurales. Il concevait celles-ci essentiellement comme des «documents" qui pouvaient éclairer les rapports entre forme et fonction et contribuer à expliquer la genèse des formes architecturales ${ }^{25}$. L'analyse systématique du passage des formes issues directement des contraintes techniques et fonctionnelles vers des formes "esthétiques ", pour lesquelles le rapport forme-fonction paraît moins évident, constitue en effet la thématique que l'architecte istrien donnait comme base de sa propre recherche sur l'architecture rurale italienne. Il l'avait commencée depuis quelques mois et elle était destinée à aboutir à la Triennale de 1936. Dans le fascicule de décembre, Pagano revient à la fin sur ce thème en identifiant la maison rurale également comme un antidote potentiel contre l'architecture grandiloquente et monumentale portée aux nues par le régime : se confronter avec sa simplicité, avec son authenticité, son essence " astylistique » et fonctionnelle, c'est, selon ses propres mots, « comme faire un régime avec des nourritures simples pour celui qui s'est rendu malade avec la pâtisserie des cariatides ${ }^{26}$ ».

12 La recherche de Pagano sur l'architecture rurale italienne trouva dans la photographie un extraordinaire instrument de documentation, inaugurant ainsi une passion destinée à se prolonger toute sa vie, comme en témoigne la richesse de ses archives photographiques ${ }^{27}$. «Pour recueillir rapidement beaucoup de matériel documentaire sur ce thème - rappelait plus tard Pagano -, [il] écarta tout de suite tous les systèmes de représentation au moyen de dessins, en raison de leur caractère trop lent, suggestif et non scientifique » et avec un 
Rolleiflex en bandoulière, il créa un «vocabulaire d'images qui parlent de l'Italie à [sa] manière ${ }^{28}$ ». Dans les mois qui précédèrent la Triennale de 1936, Pagano, "chasseur d'images ", parcourut donc la Péninsule du Nord au Sud, « pour établir sans équivoque les beautés de l'Italie rurale et pour montrer les différentes phases d'évolution de l'habitation humaine ", avec la conscience de devoir tout faire par lui-même étant donné le peu d'intérêt pour l'architecture rurale démontré à cette époque par les surintendants aux beaux-arts ${ }^{29}$.

13 Le résultat de ce travail fut l'exposition sur l'architecture rurale dans le Bassin méditerranéen, réalisée avec Guarniero Daniel dans le cadre de la $\mathrm{VI}^{\mathrm{e}}$ Triennale de Milan en 1936, ainsi que le catalogue qui l'accompagnait, destiné à illustrer l'architecture rurale italienne (fig. $\left.\mathbf{n}^{\circ} \mathbf{9}\right)$.

Figure 9

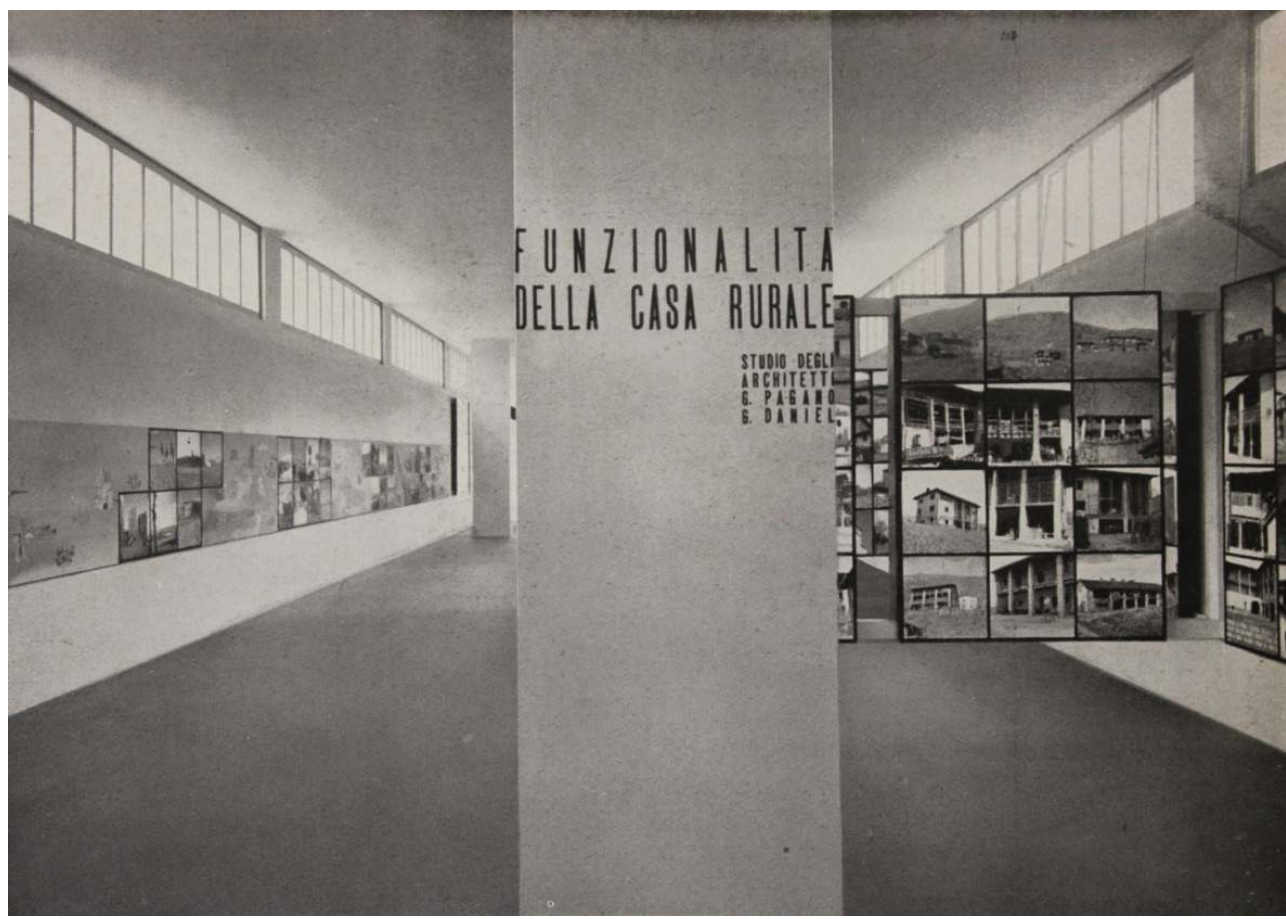

L'Exposition sur l'architecture rurale dans le Bassin méditerranéen à la Triennale de 1936. D'après Casabella, 1936, nº 104 et d'après le catalogue Architettura rurale italiana.

REPRO. D'AMIA, GIOVANNA. (c) GIOVANNA D'AMIA.

L'exposition, dont les archives de la Triennale ne conservent que quatre vues photographiques ${ }^{30}$, était montée dans le pavillon de l'Architecture réalisé sur les dessins de Pagano à côté du bâtiment principal. Le long de la paroi à gauche de l'entrée étaient disposées, en alternance avec des compositions picturales de Constantino Nivola, des photographies qui présentaient les différentes physionomies du paysage italien (littoral, basse plaine, haute plaine, colline, montagne, haute montagne, lac) auxquelles faisaient suite trois cartes géographiques qui présentaient, respectivement, les cultures agricoles, les conditions météorologiques et la nature géologique des différentes régions d'Italie.

La paroi opposée était équipée d'une série de panneaux soutenus par de frêles montants métalliques et constitués de séquences de tirages photographiques, organisés par thème et munis de brèves notices explicatives (fig. $\mathbf{n}^{\circ} \mathbf{1 0}$ ). Le parcours commençait par l'examen de la chaumière et de la cabane au toit de chaume comme système originel de couverture, 
et se poursuivait par l'étude de l'évolution des trulli, cabanes en maçonnerie de la région des Pouilles. Il reconstruisait donc le développement des couvertures depuis la voute jusqu'au toit plat. On trouvait ensuite une section consacrée aux pigeonniers, aux petites tours, aux loggias et aux escaliers extérieurs typiques de la Toscane ainsi qu'une partie qui «établit les relations entre la culture du maïs et les loggias extérieures caractéristiques de la région lombarde, de celle de Bergame et de Trente ${ }^{31} »$. Elle enquêtait sur son évolution en partant des structures en bois primitives pour arriver aux arcades en maçonnerie. Enfin, l'exposition s'achevait avec un " pilier à part » où étaient présentés des reliefs de maisons rurales de la région de Palerme, fournis par le Regio Politecnico de la cité sicilienne ${ }^{32}$.

Figure 10

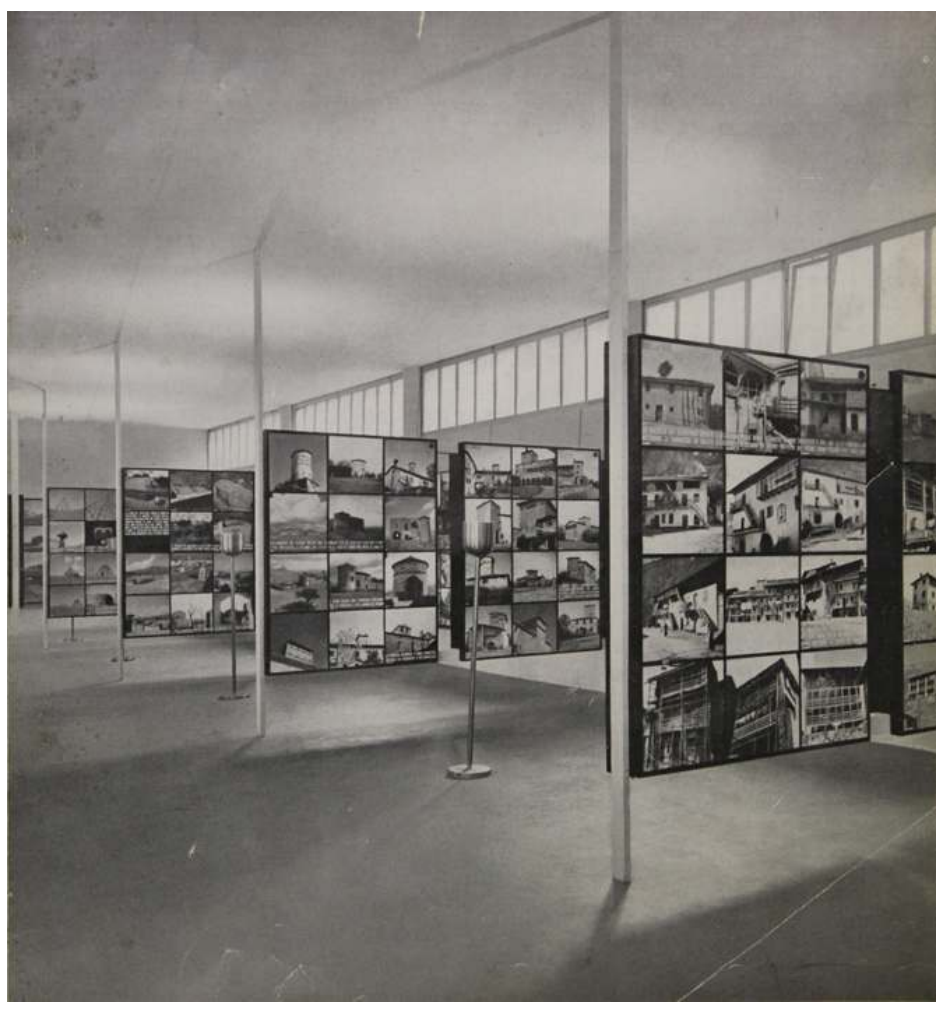

L'Exposition sur l'architecture rurale dans le Bassin méditerranéen à la Triennale de 1936. D'après Casabella, 1936, nº 104 et d'après le catalogue Architettura rurale italiana.

REPRO. D'AMIA, GIOVANNA. (c) GIOVANNA D'AMIA.

Les images photographiques qui matérialisaient l'exposition étaient en majeure partie l'œuvre de Pagano mais son objectif qui consistait à offrir un panorama des régions italiennes le plus étendu possible l'amena à se doter de collaborateurs. Pier Niccolò Berardi, membre du Groupe toscan qui en 1933 avait remporté le concours pour la gare ferroviaire de Santa Maria Novella à Florence, lui fournit vingt-quatre images de fermes toscanes $^{33}$; Roberto Pane, qui venait d'achever une recherche sur l'architecture rurale en Campanie, collabora avec des informations et des photographies d'Ischia et de Capri ${ }^{34}$; Gino Chierici, à l'époque surintendant aux monuments de la Campanie, mit à sa disposition un recueil photographique sur les trulli des Pouilles. De leur côté, les architectes Lorenzo Chiaraviglio et Angelo Scattolin apportèrent leurs contributions avec des informations sur les demeures rustiques du Latium et les toits de chaume de Vénétie ${ }^{35}$ 
Figure 11

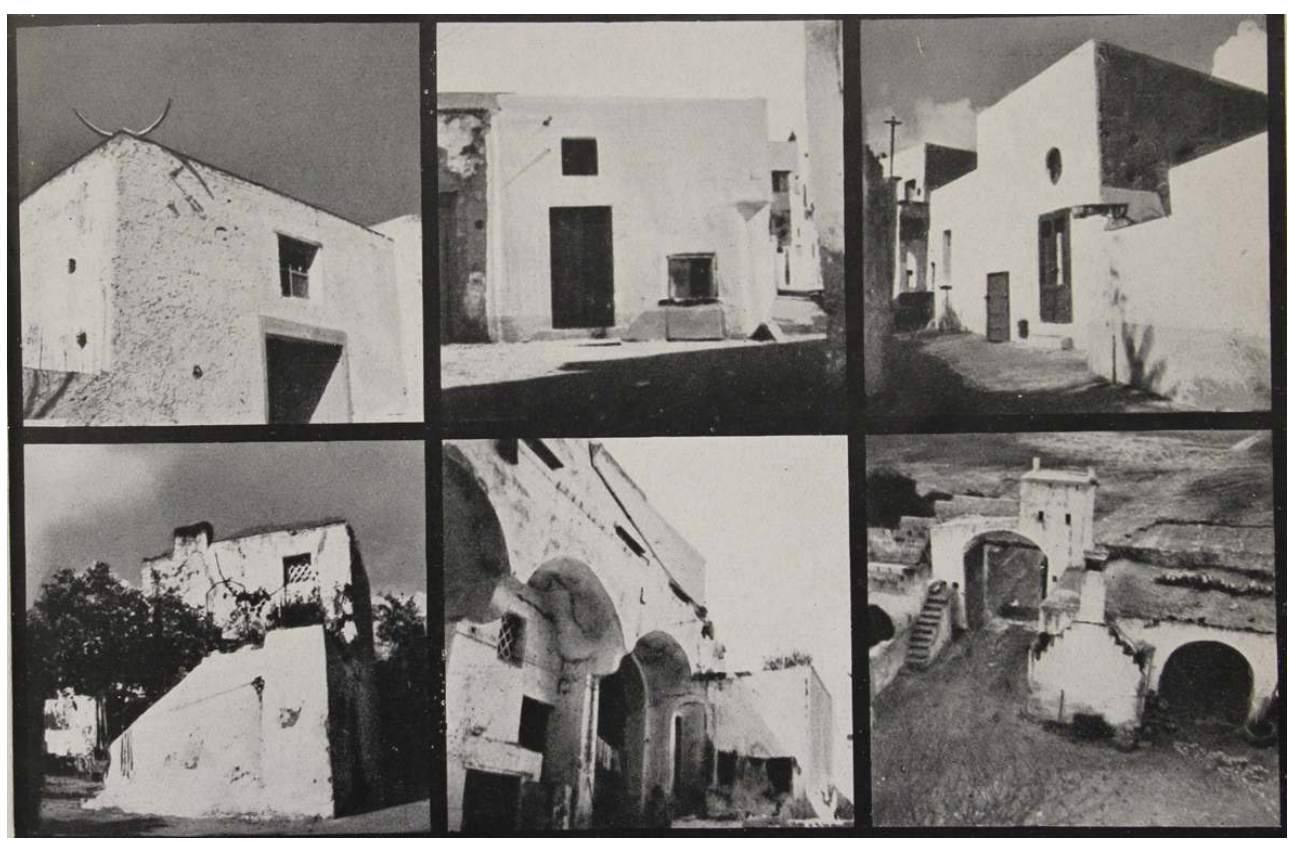

Maison à terrasse de la région de Naples. Photographie de Giuseppe Pagano et Roberto Pane. D'après le catalogue Architettura rurale italiana.

REPRO. D'AMIA, GIOVANNA. (c) GIOVANNA D'AMIA.

17 La logique qui animait l'exposition se reflète dans le catalogue imprimé, édité par Ulrico Hoepli et mis en page par Antonio Marchi. Les images photographiques s'y succèdent, comme une séquence filmique sur l'ensemble des soixante-treize pages du texte ${ }^{36}$. Là encore, les documents sont organisés sur une base essentiellement typologique, de manière à mettre en évidence la loi qui organise le développement des formes architecturales à partir d'une forme primitive découlant de la fonction à remplir, selon un rapport de cause à effet (fig. $\mathbf{n}^{\circ} \mathbf{1 1}$ ). Le schéma d'interprétation du rapport fonctionforme proposé par Pagano est l'expression d'une conception évolutionniste qui tient néanmoins compte de composants culturels, en soulignant la manière dont les formes tendent à se conserver même quand la visée utilitaire s'est éteinte ${ }^{37}$. Il l'illustre par les petites tours-belvédères des maisons toscanes, souvenir des pigeonniers originels ${ }^{38}$ (fig. $\mathbf{n}$ •12), ou par les cheminées de Vénétie dont la forme allongée constitue un héritage des couvertures de chaume des commencements ${ }^{39}$. 


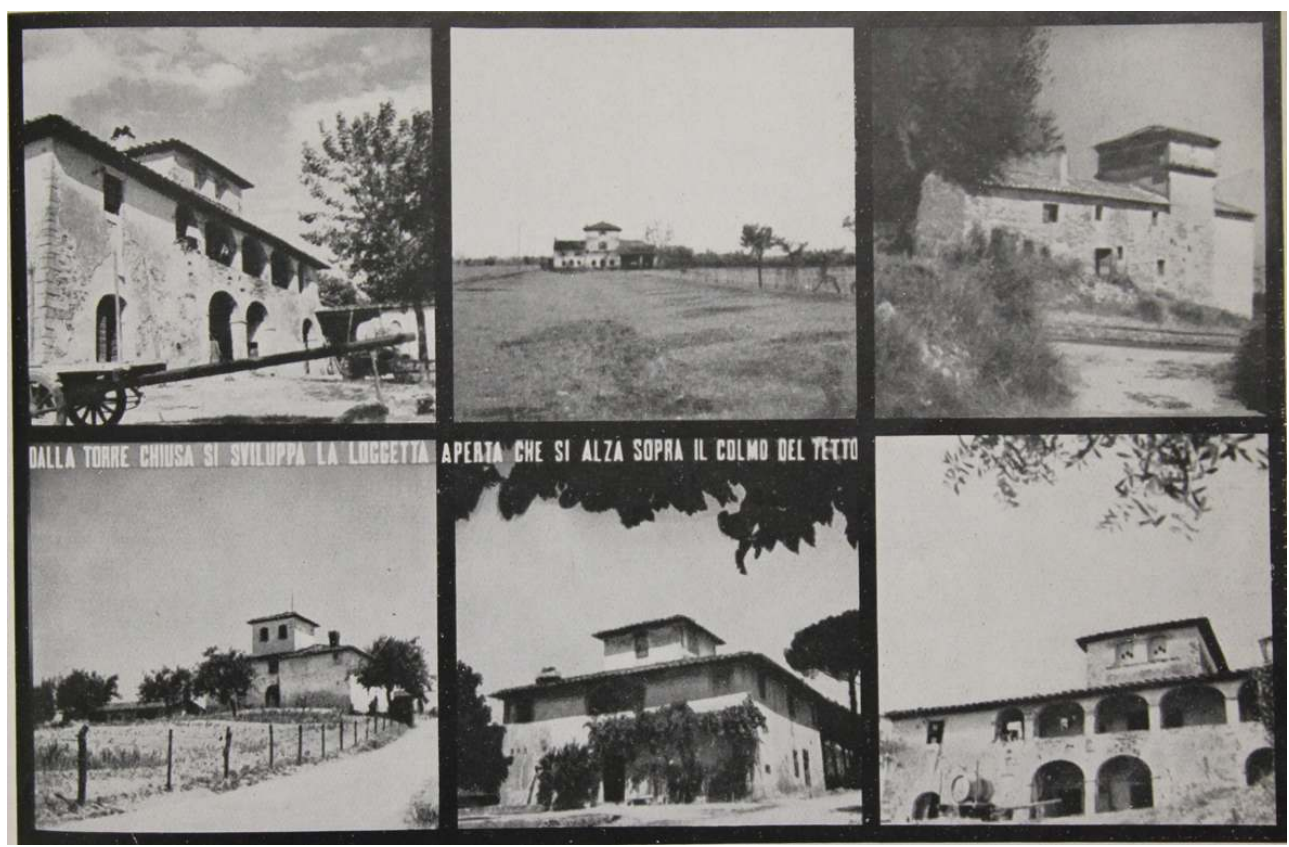

Évolution des pigeonniers en tours-belvédères. Photographie de Giuseppe Pagano. D'après le catalogue Architettura rurale italiana.

REPRO. D'AMIA, GIOVANNA. () GIOVANNA D'AMIA.

Le but de Pagano n'était pas tellement, même si c'était latent, la refondation de l'histoire de l'architecture à partir d'une loi sur l'évolution des formes ${ }^{40}$. Son objectif prioritaire était plutôt d'établir une équivalence entre architecture rurale et architecture moderne sur la base d'une dérivation commune à partir d'exigences d'ordre fonctionnel: l'architecture rurale est en effet «claire, logique, moralement mais aussi formellement très proche du goût contemporain » et l'exposition mise en place à la Triennale avait pour but déclaré de « démontrer la valeur esthétique de la fonctionnalité »"11.

19 À la lumière du but poursuivi par Pagano, le déplacement d'accent, du contexte italien au contexte méditerranéen, acquiert du sens. Il différencie la recherche sur le terrain du projet de l'exposition et ce dernier du contenu du catalogue. Le renoncement à une exposition centrée uniquement sur le contexte italien paraît en effet motivé par la volonté d'éviter l'équivoque consistant à se prévaloir de l'idée d'une voie nationale de l'architecture moderne inspirée par la tradition locale de construction. Par ailleurs, l'ouverture au Bassin méditerranéen, dans lequel la maison rurale revêt une grande variété de formes en fonction des conditions de l'environnement, contestait l'idée d'une « architecture méditerranéenne » comme tendance stylistique ${ }^{42}$. La méditerranéité, semble dire Pagano, ne consiste pas tant dans un paradigme formel (volumes purs, toits plats, etc.) que dans la correspondance avec une loi d'évolution des formes dans des contextes géographiques proches.

20 La comparaison entre les différentes régions de Méditerranée permit de réunir des compétences dans des domaines spécifiques. Collaborèrent en effet à la documentation de l'exposition : Mirko Buccianti, qui mit à disposition des informations et des photographies de maisons paysannes de Basse-Égypte ${ }^{43}$; Emilio Moya Lledós, auquel on doit les documents sur l'architecture rurale espagnole ${ }^{44}$; Gustavo Pulitzer-Finali, qui fournit des 
indications sur le Sahara sud-algérien ${ }^{45}$ (fig. $\mathbf{n}^{\circ} \mathbf{1 3}$ ), et surtout Giovanni Pellegrini, auteur en 1933 d'une habitation coloniale dans un milieu rural inspirée de l'architecture autochtone de la Lybie ${ }^{46}$, qui apporta sa contribution avec une relation documentée sur la Tripolitaine.

Figure 13

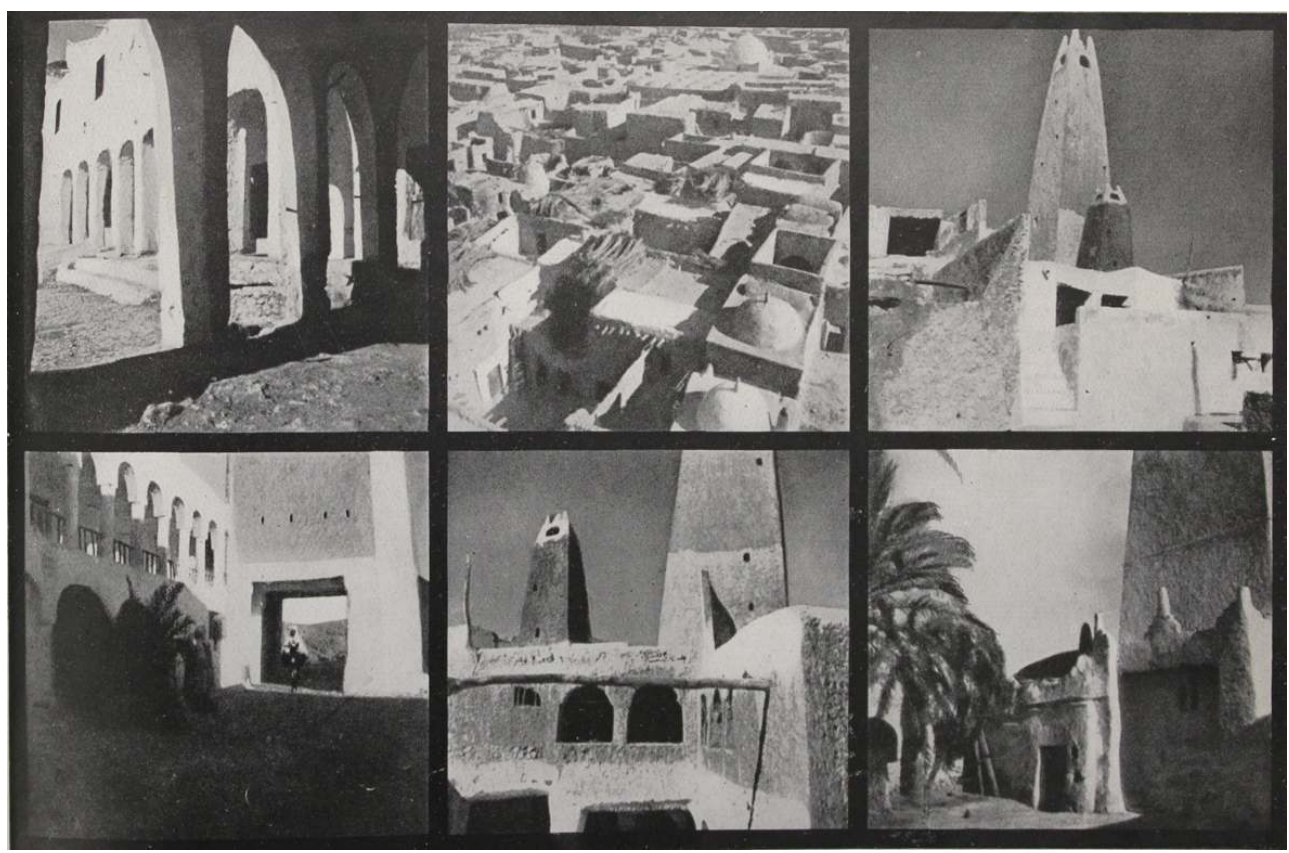

Vues de la ville des Mozabites, dans le sud de l'Algérie. Photographie de Gustavo Pulitzer-Finali (?). D'après le catalogue Architettura rurale italiana.

REPRO. D'AMIA, GIOVANNA. (C) GIOVANNA D'AMIA.

Exposition et catalogue répondaient, en définitive, à un projet culturel précis : présenter, à travers l'analyse de l'habitation rurale, un « dictionnaire de la logique constructive de l'homme» afin de balayer les préjugés d'ordre esthétique et de promouvoir une méthodologie de projet fondée sur des principes de moralité et de fonctionnalité. Il s'agit, à partir de là, de créer une architecture honnête, anonyme, exempte de préoccupations stylistiques. La preuve - pour autant qu'elle fût nécessaire - en est fournie par la correspondance entre l'exposition sur l'architecture rurale et l'« Exposition des systèmes de construction moderne » conçue par Pagano pour la même édition de la Triennale en $1936^{47}$. Un parcours qui faisait un grand usage de l'outil photographique illustrait les possibilités et les avantages de l'emploi des matériaux et des technologies les plus innovantes.

\section{Échos de l'exposition et premières réalisations}

L'exposition de Pagano à la Triennale de 1936 obtint de nombreux comptes-rendus positifs, et pas seulement dans les pages de Casabella $a^{48}$, tout en se heurtant à la perplexité du milieu des historiens et des cercles moins bienveillants à l'égard de l'architecture rationaliste ${ }^{49}$. Roberto Pane lui-même, qui avait collaboré à l'exposition, critiquait le déterminisme fonctionnaliste de Pagano dans son ouvrage sur l'architecture rurale de Campanie en niant qu'il n'y ait que des exigences pratiques au fondement de la création 
architecturale ${ }^{50}$. L'accent mis sur l'importance des facteurs d'évolution dans l'analyse de l'architecture rurale réveilla en revanche l'intérêt des géographes qui, dans les enquêtes suivantes - comme celles de Giuseppe Nangeroni -, montraient une plus grande attention à la dimension historique des phénomènes d'implantation d'habitations, considérés comme les effets d'un contexte social et économique en perpétuelle transformation ${ }^{51}$. Même les urbanistes furent sensibilisés au problème des installations rurales, qui constituèrent un thème spécifique de leur premier congrès national en $1937^{52}$.

L'autorité acquise par Pagano dans le débat interdisciplinaire se confirma par sa présence, en qualité de vice-président, au IV ${ }^{e}$ Congrès des arts et des traditions populaires qui se tint à Venise en 1940 et qui comptait une session spécifique consacrée à l'architecture rustique en Méditerranée. Dans sa relation introductive, l'ingénieur Giuseppe Ciribini insistait sur l'importance de l'étude de la genèse et de l'évolution des formes, en proposant un schéma interprétatif de la maison méditerranéenne selon un processus linéaire, depuis l'organisme monocellulaire jusqu'à l'ensemble pluricellulaire ${ }^{53}$.

Le projet de structures rurales demeura en revanche la chasse gardée des ingénieurs qui, lors du Congrès pour le développement de l'agriculture en 1938, s'accaparèrent l'étude des systèmes de construction et des types de bâtiments les plus adéquats aux exigences des entreprises agricoles. Seul Bertolazzi, dans l'introduction de ses projets, portait de nouveau un regard rétrospectif sur les caractéristiques des habitations émiliennes, en reconnaissant sa propre dette à l'égard des géographes et des architectes ${ }^{54}$.

Dans ce cadre, il faut donc signaler la maison-ferme que Lorenzo Chiaraviglio, l'un des collaborateurs de l'exposition de Pagano, conçut aux portes de Rome pour le hiérarque fasciste Roberto Farinacci. La proposition initiale, approuvée en octobre 1940, prévoyait un édifice avec une remise et un dépôt de céréales au rez-de-chaussée, des pièces d'habitation au premier étage, un grenier et un réservoir d'eau dans la tour. Elle employait des formes rationalistes, avec néanmoins un recours important à des matériaux traditionnels de l'architecture rurale romaine. La référence folklorique trop explicite avec la tour, les portiques et le patio ne pouvait pas obtenir la faveur de Pagano, en plus du fait que, dans la phase de réalisation, les finalités esthétiques finirent par l'emporter sur les exigences agricoles ${ }^{55}$. 


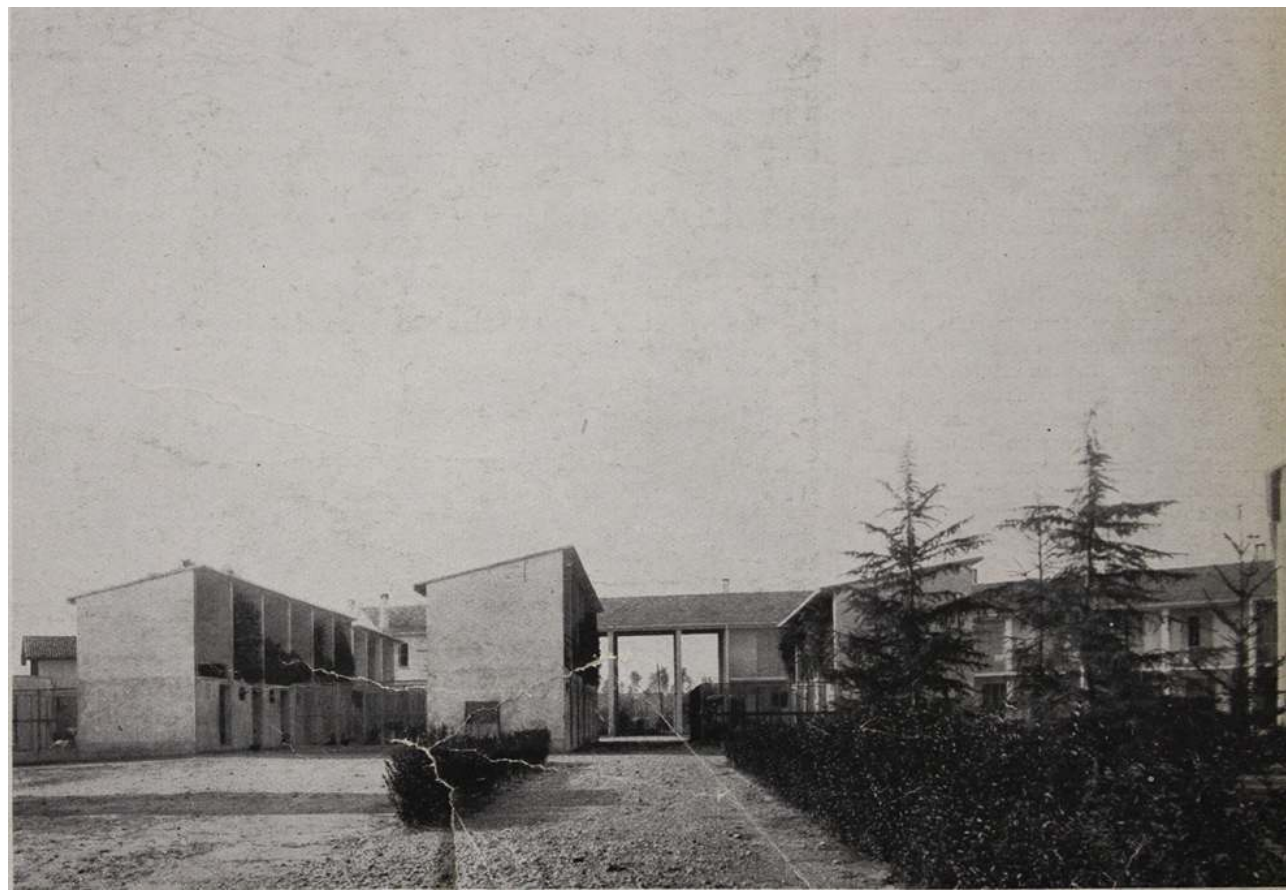

Maisons paysannes de Mario Asnago et Claudio Vender à Torrevecchia Pia. D'après Casabella, 1940, n - 146.

REPRO. D'AMIA, GIOVANNA. (C) GIOVANNA D'AMIA.

La seule construction rurale dessinée par des architectes à laquelle Pagano reconnut une valeur exemplaire se trouve en fait parmi les maisons paysannes réalisées à Torrevecchia Pia, près de Pavie, par Mario Asnago et Claudio Vender (fig. $\mathbf{n}^{\circ} \mathbf{1 4}, \mathbf{n}^{\mathbf{0}} \mathbf{1 5}$ ), qui à ses yeux "démontrent que l'on peut faire une œuvre bonne et modeste sans recourir à la rhétorique du faux et du folklore ${ }^{56} \%$. Les deux jeunes architectes se révélaient en effet attentifs aux aspects économiques et fonctionnels du problème, pas seulement à l'égard de l'habitation mais aussi pour ce qui concerne les services (grenier, porcherie, poulailler, etc.), et ils s'appuyaient sur des systèmes de construction modernes (structure en béton armé, planchers en brique de ciment), tout en démontrant la possibilité de concilier modernité et tradition du lieu dans cette nouvelle approche du type de la cascina (ferme) de Lombardie. 


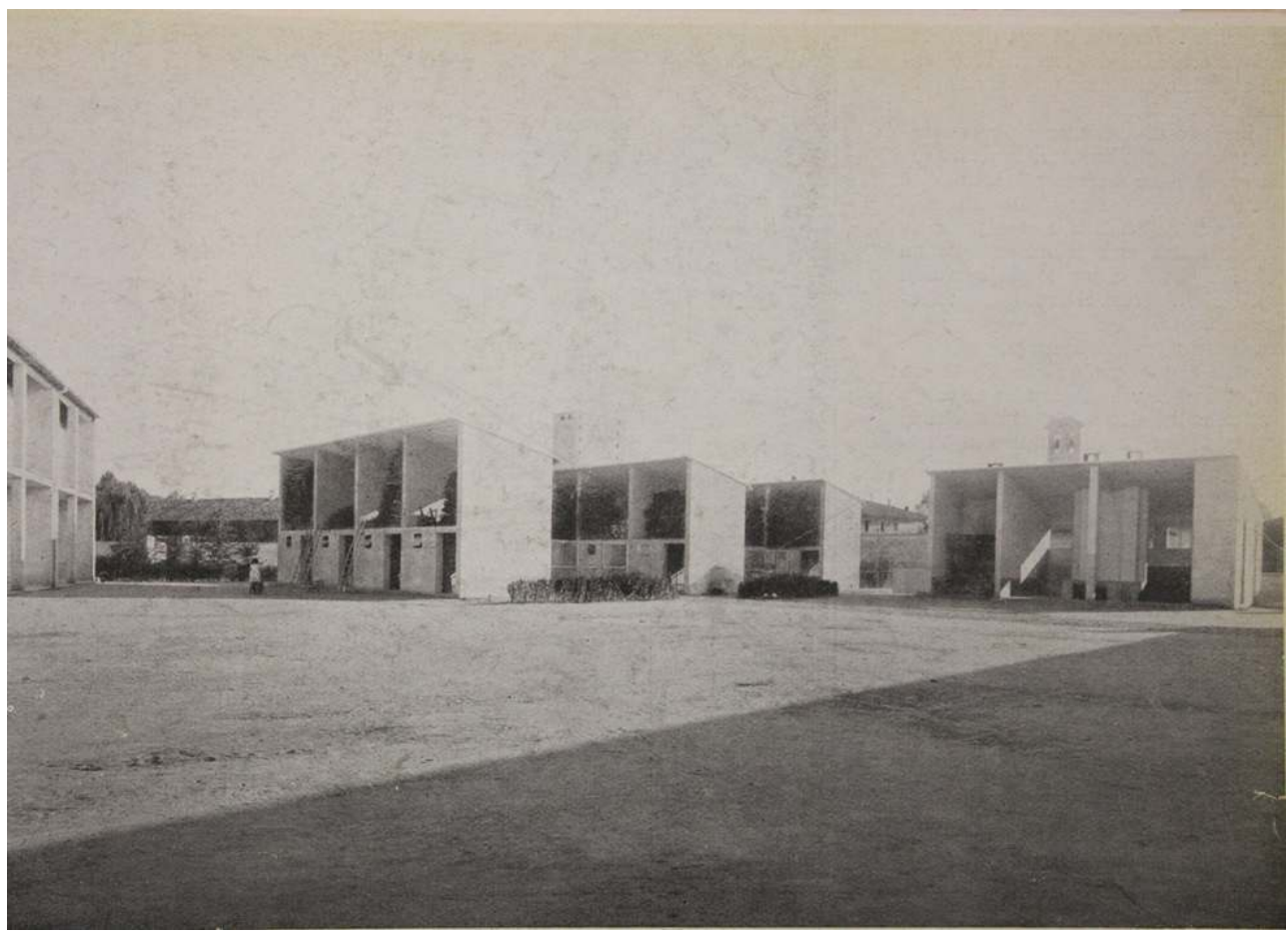

Maisons paysannes de Mario Asnago et Claudio Vender à Torrevecchia Pia. D'après Casabella, 1940, n - 146.

REPRO. D'AMIA, GIOVANNA. () GIOVANNA D'AMIA.

Une occasion de rencontre entre culture architecturale et constructions rurales aurait pu être, à cette époque, le concours pour études et projets sur les maisons rurales lancé par la Triennale de Milan en janvier 1943, en prévision de la VIII Triennale ${ }^{57}$, mais l'engagement de l'Italie dans la Seconde Guerre mondiale interrompit bientôt cette démarche. Pour mesurer l'influence de l'exposition de Giuseppe Pagano, il faut donc attendre la période de l'après-guerre, lorsque les architectes italiens, nourris par l'expérience du Razionalismo, se confrontèrent à une dimension régionaliste et au genius loci.

\section{NOTES}

1. - Traduit de l'italien par Gérald Larché.

2. - La Rivista geografica italiana avait été fondée à Florence en 1894 par Filippo Maria Pasanisi et, dès 1895 , elle était devenue l'organe de la Società di studi geografici e coloniali (Societé d'études géographiques et coloniales).

3. - Voir DEMANGEON, Albert. "L'habitation rurale en France. Essai de classification des principaux types ». Dans Annales de géographie, 1920, t. 29, n 161, p. 352-375. Autour du géographe 
français, voir WOLFF, Denis. " Demangeon Albert ». Dans LEVY, Jacques. Dictionnaire de la géographie et de l'espace des sociétés. Paris : Belin, 2003, p. 234-236.

4. - Parmi les premières publications du géographe italien concernant les habitations rurales, notons BIASUTTI, Renato, « Per un'inchiesta sui tipi dell'abitazione rurale in Italia ». Dans Atti del IX Congresso geografico italiano, Gênes : 1925, p. 205-206 ; id., « Per lo studio dell'abitazione rurale in Italia ». Dans Rivista geografica italiana, XXXIII (1926), p. 1-24. Renato Biasutti est aussi l'auteur du premier volume (La casa rurale della Toscana. Bologne: 1938) d'une collection consacrée aux maisons rurales des différentes régions italiennes qui fut publiée par les soins $\mathrm{du}$ Comitato nazionale geografico jusqu'en 1946, puis par le Consiglio nazionale delle ricerche.

5. - Voir BIASUTTI, Renato. «Insediamenti agricoli ed abitazioni rurali in Italia ». Dans Atti del X Congresso Geografico Italiano. Milan : 1927, p. 241-246.

6. - Voir ALGRANATI, Gina. «Il folclore e la geografia. Estensione e limiti ». Dans Atti del X Congresso Geografico Italiano (citation p. 259-263) ; id., « Osservazioni geografiche sull'architettura rustica ». Rivista geografica italiana, XXXIX (1932), p. 35-38.

7. - Bruno Nice était engagé dans une recherche qui aboutira à la publication du second volume de la collection sur les habitations rurales italiennes lancée par le Comité national géographique (Voir NICE, Bruno. La casa rurale nella Venezia Giulia. Bologne : Comitato geografico italiano, 1940).

8. - Voir surtout, à ce propos, les recherches d'Aldobrandino Mochi pour la réalisation d'un Musée national d'anthropologie et d'ethnologie à Florence au début du XIX ${ }^{\mathrm{e}}$ siècle.

9. - Voir BROCHEREL, Giulio. «La casa rustica valdostana ». Dans Atti del III Congresso di Arti e tradizioni popolari, Rome, Edizioni OND, 1936, p. 197-200.

10. - Voir «Atti del Comitato per le Arti popolari». Lares, 1936, p. 219-229. Le questionnaire examinait tous les témoignages matériels, depuis les habitations et les établissements ruraux jusqu'aux outils domestiques et agricoles, dans le but d'aider pour le tri des objets destinés aux expositions et aux musées d'Art populaire.

11. - La revue Lares, voix de la Società di Etnografia italiana fondée en 1912 par Lamberto Loria, favorisait la rencontre entre les savants de différents domaines. Parmi les interventions sur la maison rurale, voir surtout ANGELINI, Luigi. "Aspetti dell'architettura rustica nelle valli bergamasche ». Dans Lares, 1932, p. 77-81. FORMENTINI, Ubaldo. « Note sull'architettura rustica nella Liguria Orientale ». Lares, 1937, p. 101-105.

12. - GRIFFINI, Enrico. "La casa rustica nella Valle Gardena ». Architettura e arti decorative, IV (1924-1925), vol. II, p. 291-298 ; GEROLA, Giuseppe. "Architettura minore e rustica trentina ». Architettura e arti decorative, VIII (1928-1929), vol. II, p.291-301. La revue Architettura e arti decorative, fondée par Gustavo Giovannoni et Marcello Piacentini en 1921, fut dès 1927-1928 l'organe officiel du Sindacato Nazionale Fascista Architetti, sous la direction d'Alberto Calza-Bini. En 1931 elle changea de nom et devint Architettura, dirigée par Marcello Piacentini.

13. - Voir PANE, Roberto. "Tipi di architettura rustica in Napoli e nei Campi Flegrei ». Dans Architettura e arti decorative, VII (1927-1928), vol. II, p. 529-543. MARCONI, Plinio. «Architetture minime mediterranee e architettura moderna ». Architettura e arti decorative, IX (1929-1930), vol. I, p. 27-44. La querelle sur l'origine des toits à voûte dans la région du Vésuve impliqua différents savants. Plinio Marconi soutenait l'hypothèse d'une influence arabo-normande, tout comme Edwin Cerio (voir La casa nel paesaggio di Capri. Naples : 1922), au contraire de Renato Biasutti, qui défendait une origine gréco-byzantine.

14. - BERLAM, Arduino. "Case caratteristiche della Carnia ». Rassegna di architettura, II (1930), p. 153-160. Rassegna di architettura, fondée en 1929, était dirigée par Giovanni Rocco.

15. - TINTI, Mario. «L'equivoco dell'architettura rustica ». Casabella, janvier 1933, p. 51-52. Mario Tinti est aussi l'auteur du livre L'Architettura delle case coloniche in Toscana, édité à Florence en 1934 et illustré avec 32 dessins du peintre Ottone Rosai. Dans le compte rendu de ce livre, paru dans L'Ambrosiano du 10 octobre 1935, Carlo Carrà souligne que "Tinti rileva la perfetta integrazione al clima e alle funzioni che fa della casa rurale un prodotto quasi senza tempo e 
senza stile e nota come tali principi siano stati riadattati dagli architetti razionalisti per sottrarsi alla retorica degli stili e delle facciate» («Tinti note l'adaptation parfaite au climat et aux fonctions, ce qui fait de la maison rurale un produit quasiment intemporel et dépourvu de style et il remarque que des principes similaires ont été réadaptés par les architectes rationalistes en vue de se soustraire à la rhétorique des styles et des façades »).

16. - RAVA, Carlo Enrico. «Di un'architettura coloniale moderna ». Domus, ${ }^{\circ} 41,1931$, p. 39-43 et 89, $\mathrm{n}^{\circ} 42$, p. 32-36. Rappelons que Domus avait été fondé par Gio Ponti en 1928. Sur Rava, voir FULLER, Mia, « Carlo Enrico Rava. The radical first formulations of colonial rationalism ». A.A.R.P. Environmental Design, 1994-1995, 1-2, p. 150-159.

17. - MICHELUCCI, Giovanni. «Fonti della moderna architettura italiana ». Domus, 1932, $\mathrm{n}^{\circ} 56$, p. $460-461$.

18. - PERESSUTTI, Enrico. «Architettura mediterranea ». Quadrante, 1935, n 21, p. 40. La revue, fondée en 1933, était dirigée par Pier Maria Bardi et Massimo Bontempelli et soutenait une "tendance dans la tendance», à la fois italienne et méditerranéenne à l'intérieur de l'architecture moderne. Pour un cadre d'ensemble sur le débat concernant la méditerranéité, voir notamment PATETTA, Luciano (dir.), L'Architettura in Italia 1919-1943. Le polemiche. Milan : Clup, 1972. DANESI, Silvia. « Aporie dell'architettura italiana in periodo fascista, mediterraneità e purismo ». Dans PATETTA, Luciano, DANESI, Silvia (dir.). Il razionalismo e l'architettura in Italia durante il fascismo. Venise : Electa, 1976, p. 21-28. GRAVAGNUOLO, Benedetto. Il mito mediterraneo nell'architettura contemporanea. Naples : Electa, 1994.

19. - À ce propos voir notamment : collectif. Metafisica costruita. Le città di fondazione degli anni Trenta dall'Italia all'oltremare. Milan : Touring Club Italiano, 2002. Pour ce qui concerne les villages ruraux dans les colonies italiennes: GRESLERI, Giuliano, MASSARETTI, Pier Giorgio, ZAGNONI, Stefano (dir.). Architettura italiana d'oltremare 1870-1940. Venise : Marsilio, 1993. CRESTI, Franco. «Edilizia e urbanistica nella colonizzazione agraria della Libia (1922-1940)». Storia Urbana, $\mathrm{n}^{\circ} 40$, 1987, p. 189-231. CAPRESI, Vittoria, L'utopia costruita. Guida all'architettura dei centri rurali fascisti in Libia (1934-1940). Bologne : BUP, 2011.

20. - Parmi les congrès rappelons le premier Convegno lombardo di igiene rurale (Milano, 1930), le II ${ }^{\mathrm{e}}$ Congresso internazionale di tecnica sanitaria e di igiene urbana (Milano, 1931), et surtout le III Convegno nazionale degli ingegneri italiani (Trieste, 1935) portant sur le règlement de construction pour les maisons rurales, la standardisation et l'aménagement des établissements ruraux et semi-ruraux. Parmi les concours, le plus intéressant est celui lancé par le Sindacato Fascista Ingegneri della Provincia di Milano en 1934. Pour ce qui concerne, enfin, les revues spécialisées, voir surtout La Casa (dès 1918), L’Ingegnere (revue du Sindacato nazionale fascista ingegneri, dès 1927), et le Bollettino dei tecnici agricoli (dès 1936), qui en 1939 devint Rivista di estimo agrario e genio rurale et qui, en 1940, publia un dossier monographique sur la question des maisons rurales.

21. - CIOCCA, Gaetano. "Progetto di casa rurale». Quadrante, $n^{\circ} 26,1935$, p. 6-27. Le modèle proposé, destiné à la production en série, examinait les problèmes économiques, hygiéniques et fonctionnels de la maison rurale, tout en proposant une solution architecturale en forte rupture par rapport à la tradition.

22. - Voir PAGANO, Giuseppe. «Case rurali ». Dans Casabella, nº 86, 1935, p. 8-15.

23. - Sur l'architecte italien et son activité à la rédaction de Casabella, voir surtout: ALBINI, Franco, PALANTI, Giancarlo, CASTELLI, Anna (dir.). Giuseppe Pagano Pogatschnig: architetture e scritti. Milan : Editoriale Domus, 1947 (réimpression par Casabella, $\mathrm{n}^{\circ} 763$, Milan, 2008). DE SETA, Cesare. Giuseppe Pagano, Architettura e città durante il fascismo. Rome-Bari : Laterza, 1978. SAGGIO, Antonino. L'Opera di Giuseppe Pagano tra politica e architettura. Bari : Dedalo, 1984. DE SETA, Cesare. Il destino dell'architettura : Persico, Giolli, Pagano. Rome-Bari : Laterza, 1985.

24. - PAGANO, Giuseppe. « Case rurali ». Casabella, nº 86, 1935. 
25. - Voir PAGANO, Giuseppe. «Documenti di architettura rurale». Casabella, $\mathrm{n}^{\circ}$ 95, 1935, p. 18-25 : «La genesi della forma architettonica è dovuta al soddisfacimento di esigenze tecniche e funzionali, conosciamo più per intuizione che per esperienza che una forma naturalmente estetica è stata inizialmente suggerita dalla risoluzione di una necessità tecnica o funzionale. Ma i rapporti tra l'ultimo anello della catena e quello iniziale spesso ci sfuggono perché crediamo morte o disperse nella preistoria quelle testimonianze che han servito da lievito alla rappresentazione aulica ». («La genèse des formes architecturales résulte de leur conformité à des exigences d'ordre technique et fonctionnel, et nous savons plus par intuition que par expérience qu'une forme naturellement esthétique a été suggérée initialement par la résolution d'une nécessité technique ou fonctionnelle. Mais les rapports entre le dernier maillon de la chaîne et le premier nous échappent souvent parce que nous croyons que ces témoignages qui ont servi de levain pour la représentation raffinée sont morts ou dispersés dans la préhistoire. ») 26. - PAGANO, Giuseppe. «Architettura rurale italiana ». Casabella, nº 96, 1935, p. 16-23.

27. - À ce propos, voir DE SETA, Cesare (dir.). Giuseppe Pagano fotografo. Milan: Electa, 1979. Giuseppe Pagano. Vocabulario de imagenes (catalogue d'exposition). Valence : Lampreave \& Millan, 2008. Notamment DE SETA, Daria. «Giuseppe Pagano fotografo. Architettura rurale italiana. VI Triennale, Milano, 1936 ». Dans El GATCPAC y su tiempo. Politica, cultura y arquitectura en los años treinta, actes du $\mathrm{V}^{\mathrm{e}}$ Congrès du Do.co.mo.mo.. Barcelone : Fundaciòn Do.co.mo.mo., 2005.

28. - PAGANO, Giuseppe. «Un cacciatore di immagini ». Cinema, décembre 1938. ALBINI, Franco, PALANTI, Giancarlo, CASTELLI, Anna (dir.). Giuseppe Pagano Pogatschnig: architetture e scritti. Milan : Editoriale Domus, 1947 (réimpression par Casabella, nº 763, Milan, 2008), p. 67-68.

29. - Voir PAGANO, Giuseppe. «Un cacciatore di immagini ». Cinema, décembre 1938: "Con l'egida della Triennale di Milano mi rivolsi così a tutti i regi sopraintendenti d'Italia pregandoli di fotografare, spese pagate, i cascinali e le case rurali e i villaggi più interessanti della loro regione. Le risposte che allora mi pervennero, scoraggianti, assurde, inverosimili, mi convinsero che dovevo assolutamente fare tutto da me [...] quando persino la sopraintendenza della Toscana mi rispondeva che 'in quelle campagne non c'era niente di interessante da fotografare.» («Sous l'égide de la Triennale de Milan je me tournai ainsi vers tous les superintendants royaux d'Italie en les priant de photographier, tous frais payés, les fermes et les maisons rurales ainsi que les villages les plus intéressants de leurs régions. Les réponses qui me parvinrent alors, décourageantes, absurdes, invraisemblables, me convainquirent que je devais absolument tout faire par moi-même [...] lorsque même la surintendance de la Toscane me répondit que "dans ces campagnes il n'y avait rien d'intéressant à photographier". »)

30. - Voir Archivio Storico e Fotografico de la Triennale, Milan, TRN VI - 04- 0269, 0273, 0274 e 0275. Les photographies sont réalisées par le Stabilimento Fototecnico S.A. Crimella.

31. - Guida della Sesta Triennale. Milan : Società Anonima Milanese Editrice, 1936, p. 62-63.

32. - Voir à ce propos AIROLDI, Maria. «L'architettura rurale e la Triennale di Milano del '36". Dans Hevelius' webzine, octobre 1936. Le groupe sicilien, dont faisaient partie Edoardo Caracciolo, Pietro Airoldi et Vincenzo Lanza, réalisa en 1938 une exposition, avec catalogue, intitulée Rilievi di edilizia minore siciliana (Palerme, 1938).

33. - Voir à ce propos FANELLI, Giovanni, MAZZA, Barbara. La casa colonica in Toscana. Le fotografie di Pier Niccolò Berardi alla Triennale del 1936. Florence : Octavo, 1999. Sur Berardi, élève de Giovanni Michelucci, voir Pier Niccolò Berardi. Florence : Sansoni, 1973. Pier Niccolò Berardi Architetto. Milan : Mondadori, 1988. GHELLI, Cecilia. «Pier Niccolò Berardi ». Dans INSABATO, Elisabetta, GHELLI, Cecilia (dir.). Guida agli archivi di architetti e ingegneri del Novecento in Toscana. Florence : Edifir, 2007, p. 60-64.

34. - Voir PANE, Roberto. Architettura rurale campana. Florence : Rinascimento del libro, 1936. Pour plus d'informations sur l'architecte et historien napolitain, voir CASIELLO, Stella, PANE, Andrea, RUSSO, Valentina. Roberto Pane tra storia e restauro : architettura, città, paesaggio. Venise : Marsilio, 2010. 
35. - Voir Guida della Sesta Triennale. Milan : Società Anonima Milanese Editrice, 1936, p. 62-63.

36. - PAGANO, Giuseppe, DANIEL, Guarniero. Architettura rurale italiana. Milan : Hoepli, 1936. Aux pages composées de texte et d'images - celles-ci sur un fond noir, selon une solution graphique fréquente dans Casabella - font suite soixante-douze photographies à pleine page, choisies parmi les deux mille environ, réalisées par Pagano e Daniel.

37. - Voir PAGANO, Giuseppe, DANIEL, Guarniero. Architettura rurale italiana. Milan : Hoepli, 1936, p. 27-28: «L'inerzia dell'uomo (che si chiama tradizione o eredità) tende effettivamente a conservare la forma anche quando lo scopo utilitario e primario ha cessato di esistere. La forma, ormai divenuta puramente estetica, rimane come un'aggiunta ornamentale che non ricorda spesso nemmeno lontanamente la sua origine primitiva. Così il nostro studio analitico ha cercato di scoprire per ogni cosa, per ogni forma la sua origine, la sua iniziale causa utilitaria e si è potuto costatare che mai la fantasia dell'uomo ha creato senza uno scopo e senza una ragione logica quelle forme tanto sorprendenti quando esse vengono osservate in sede estetica. » ( L'inertie de l'homme (qu'on l'appelle tradition ou hérédité) tend effectivement à conserver la forme même lorsque le but utilitaire ou primaire a cessé d'exister. La forme, devenue désormais purement esthétique, demeure comme un ajout ornemental qui ne rappelle souvent même pas de loin son origine première. Ainsi notre étude analytique a cherché à découvrir pour chaque chose, pour chaque forme son origine, sa cause utilitaire initiale, et on a pu constater que jamais la fantaisie humaine n'a créé sans viser un but et sans une raison logique ces formes si surprenantes lorsqu'elles sont observées dans un lieu esthétique. »)

38. - Sur le même sujet, Daniel écrira l'année suivante un article sur la revue de l'éditeur Hoepli : DANIEL, Guarniero. « Breve storia della colombaia ». Sapere, 1937, vol. V.

39. - Voir aussi, à ce propos, PAGANO, Giuseppe. «I camini del Veneto ». Casabella, 1943, avrilmai, p. 82-85.

40. - Voir PAGANO, Giuseppe, DANIEL, Guarniero. Architettura rurale italiana. Milan : Hoepli, 1936, p. 7 : « Nella storia dell'architettura noi studiamo generalmente la storia del gusto architettonico attraverso le forme auliche adottate dalle costruzioni maggiori : i templi le chiese i palazzi. Quali ragioni tecniche, quali rapporti di tradizione formale e quali influenze di carattere economico e funzionale abbiano originato queste manifestazioni non interessano per lo più né il mondo degli studiosi né quello degli artisti. » («Dans l'histoire de l'architecture nous étudions généralement l'histoire du goût architectural à travers les formes raffinées adoptées par les constructions les plus importantes: les temples, les églises, les palais. Les raisons techniques, les rapports de traditions formelles et les influences de nature économique qui ont engendré ces manifestations, voilà qui n'intéresse en rien le monde des savants ni celui des artistes. »)

41. - Ibid., p. 6, où Pagano poursuit : « La conoscenza delle leggi di funzionalità [...] ci preserverà forse dalle ricadute accademiche, ci immunizzerà contro la retorica ampollosa e soprattutto ci darà l'orgoglio di conoscere la vera tradizione autoctona dell'architettura italiana: chiara, logica, lineare, moralmente ed anche formalmente vicinissima al gusto contemporaneo.» («La connaissance des lois de fonctionnalité [...] nous préservera peut-être des rechutes dans l'académisme, nous immunisera contre la rhétorique ampoulée et surtout nous donnera l'ambition de connaître la véritable tradition autochtone italienne : claire, logique, moralement et même formellement très proche du goût contemporain. »)

42. - Une critique à l'architecture méditerranéenne proposée par les architectes réunis autour de la revue Quadrante, avait déjà été lancée par Edoardo Persico, collaborateur de Pagano à Casabella. Voir notamment PERSICO, Edoardo. «Punto e da capo per l'architettura». Domus, novembre 1934, p. 1-9.

43. - Pour la liste des collaborateurs de Pagano, voir PAGANO, Giuseppe, DANIEL Guarniero. Architettura rurale italiana. Milan: Hoepli, 1936, p.6. Les informations sur l'activité de Mirko Buccianti en Égypte sont rarissimes. Milva Giacomelli signale sa collaboration avec Paolo Caccia Dominioni dans le projet de concours pour le champ de sport italien au Caire en 1937. Voir 
GIACOMELLI, Milva. «Clemente Busiri Vici e il rinnovato volo dell'architettura italiana in Egitto negli anni trenta». Dans GODOLI Ezio, GIACOMELLI, Milva (dir.). Architetti e ingegneri italiani in Egitto dal diciannovesimo al ventunesimo secolo. Florence : Maschietto, 2008, p. 161-183.

44. - Dès janvier 1936, Emilio Moya Lledós était directeur de l'Academia Espanola de Bellas Artes di Roma et il était aussi commissaire de la section espagnole à la $\mathrm{VI}^{\mathrm{e}}$ Triennale. Voir ESTEBAN, José. « Emilio Moya Lledós, arquitecto conservador de monumentos (1929-1936) ». Dans Roma y la tradición de lo nuevo. Diez artistas en el Gianicolo (1923-1927). Madrid : Seacex, 2003.

45. - Gustavo Pulitzer-Finali était aussi présent à la VI ${ }^{\mathrm{e}}$ Triennale de 1936 avec un projet d'ameublement de paquebot. Sur son activité en ce champ, voir PULITZER-FINALI, Gustavo. Navie Case. Architetture interne 1930-1935. Milan : Hoepli, 1935. Et RICCESI, Donato. Gustavo Pulitzer Finali. Il disegno della nave. Allestimenti interni 1925-1967. Venise : Marsilio, 1985.

46. - L'habitation coloniale en milieu rural avait été présentée à la Triennale de 1933. Sur l'activité de Pellegrini en Lybie, visant à concilier esprit fonctionnaliste et tradition autochtone, voir D 'AMIA, Giovanna. «The Work of Giovanni Pellegrini in Libya ». Dans The Presence of Italian Architects in Mediterranean Countries. Florence: Maschietto, 2008, p. 78-89; id., "L'urbanistica coloniale di Giovanni Pellegrini e la pianificazione dei villaggi libici ». Territorio, 2011, $n^{\circ} 57$, p. 125-134.

47. - L' «Exposition des systèmes de construction moderne » était réalisée par Pagano avec la collaboration de G. Frette, T. Bussi, M. Casale, P. Chiolini, A. Goldstein-Bolocan, F. Marescotti et G. G. Prevedal. Voir le compte-rendu de TEDESCHI, Enrico. «La mostra dei sistemi costruttivi moderni e dei materiali da costruzione alla VI Triennale di Milano ». Architettura, XVI, 1937, 1, p. 41-51.

48. - Voir PODESTÀ, A. « La VI Triennale, mostre di Architettura ». Casabella, nº 104, 1936, p. 8-11. CARLI, Enzo. «Il genere architettura rurale e il funzionalismo ». Casabella, n ${ }^{\circ} 107,1936$, p. 6-7. Pour Podestà, l'exposition ouvre « un nuovo campo di indagine sulla essenza ed i rapporti di necessità del fenomeno costruttivo "; tandis que Carli intervient dans la "polemica sull'architettura nazionale » au-delà des préjugés d'ordre esthétique, tout en soulignant que « la beauté spontanée de la maison rurale » («spontanea bellezza della casa rurale ») est le reflet du fonctionnalisme entendu comme norme éthique.

49. - Voir à ce propos le compte-rendu de Guglielmo De Angeli d'Ossat dans Palladio (1939, p. 40), qui trouve « insatisfaisant le traitement du point de vue historico-critique car la complexité du thème ne peut pas se résoudre à l'enchaînement trop idéal qui est imaginé » (« insoddisfacente la trattazione dal punto di vista storico-critico giacché la complessità del tema non può venire risolta secondo la troppo ideale successione immaginata »). La revue d'histoire de l'architecture Palladio avait été fondée par Gustavo Giovannoni en 1937. Parmi les articles concernant l'architecture rurale, rappelons ceux de deux collaborateurs de Pagano lors de l'exposition du 1936 : CHIARAVIGLIO, Lorenzo. «Case di Priverno ». Palladio, 1937, vol. II, p. 71. PANE, Roberto. « Appunti di architettura minore : i dintorni di Napoli ». Palladio, 1938, vol. V, p. 184-186.

50. - PANE, Roberto. Architettura rurale campana. Florence: Rinascimento del libro, 1936. Pane prend part au débat sur l'origine des couvertures voûtées, niant l'influence arabe en faveur de celle gréco-byzantine et proposant une dérivation romaine pour les maisons à cour. Les critiques de la théorie de Pagano seront reprises dans les publications de l'après-guerre (Capri, Naples, 1954 et Capri, mura e volte, Naples, 1965).

51. - Professeur de géographie à l'Università Cattolica de Milan, Nangeroni avait publié une recherche sur les centres ruraux de la province de Varèse en 1934. Dès les années 1940, il lança une collection de guides sur les établissements ruraux avec la collaboration de Giuseppe Ciribini.

52. - Voir Atti del Primo Congresso nazionale d'urbanistica. Rome : Istituto Nazionale di Urbanistica (1938).

53. - Voir CIRIBINI, Giuseppe. «Studi tecnico-estetici sull'architettura mediterranea, con particolare riguardo a quella italiana». Dans Atti del IV Congresso di arti e tradizioni popolari, 
Venise: 1940, p.573-574. Ciribini identifie une «cellule élémentaire » dans la maison méditerranéenne qui évolue avec une tendance au perfectionnement (Pagano autrement parlait d'« inertie» dans la conservation des formes), pas seulement sous l'effet de nécessités climatiques et fonctionnelles, mais selon les caractéristiques des différents peuples. Son schéma d'évolution est repris dans ses œuvres ultérieures, notamment dans CIRIBINI, Giuseppe. La casa rustica nelle valli del Rosa. Milan : Centro Nazionale Universitario di Studi Alpini, 1943.

54. - Voir BERTOLAZZI, Francesco. «L'edilizia rurale in Emilia». Dans Atti del convegno degli ingegneri per il potenziamento dell'agricoltura ai fini autarchici : Lombardia, Emilia, Tre Venezie, Piemonte . Milan : Archetipografia, 1938-1939, vol. I, p. 129-138.

55. - La villa Farinacci, autrefois centre du domaine agricole Aguzzano, est aujourd'hui propriété de la mairie de Rome qui vient de la rétablir.

56. - PAGANO, Giuseppe. "Case coloniche nella pianura lombarda ». Casabella, $n^{\circ} 146,1940$, p. 25-27. Voir aussi ZUCCHI, Cino, CADEO, Francesca, LATTUADA, Monica. Asnago e Vender. Architetture e progetti 1925-1970. Milan : Skira, 1999, notamment p. 76-79.

57. - Voir l'avis de concours dans les archives de la Triennale de Milan, et l'article « Un concorso per studi e progetti sulla casa rurale indetto dalla Triennale di Milano ». Rivista di estimo agrario $e$ genio rurale, 1943, p. 44.

\section{RÉSUMÉS}

Dans l'Italie des années 1930, l'architecture rurale fait l'objet d'un débat dont sont partie prenante des ethnographes, des géographes, des architectes et des urbanistes. C'est la politique de réorganisation agraire du régime qui suscite l'intérêt pour ce thème, mais la réflexion est aussi liée à la recherche d'une approche italienne de l'architecture moderne. C'est dans ce cadre que s'inscrit l'exposition organisée par Giuseppe Pagano pour la $\mathrm{VI}^{\mathrm{e}}$ Triennale, dans le but de démontrer la valeur esthétique de la fonctionnalité et d'enquêter sur les rapports de cause à effet qui déterminent la forme architecturale.

In Italy, in the 30s, rural architecture was the principal subject of a debate between ethnologists, geographers, architects and town planners. The fascist politics of the agrarian reorganization aroused interest in this topic, but the intellectual reflection was also stimulated by the research of an Italian modern architecture approach to the problem. In this context Giuseppe Pagano organized the 6th Triennale's exposition in order to show the aesthetic value of the functionality and to investigate the relationship of cause and effect that determine the architectural form.

\section{INDEX}

Mots-clés : architecture rurale, Giuseppe Pagano, Triennale Milano

\section{AUTEUR}

\section{GIOVANNA D'AMIA}

Chercheur en histoire de l'architecture, Politecnico di Milan giovanna.damia@polimi.it 